\title{
Frequency response characteristic of sympathetic mediated low-frequency blood pressure fluctuations in conscious rats
}

\author{
Ruei-Feng Chen ${ }^{\text {a }}$, Wan-Ting Zeng ${ }^{\text {a }}$, Chen-Tung Yen ${ }^{\text {a }}$, Meng-Li Tsai ${ }^{\text {b,* }}$ \\ ${ }^{a}$ Institute of Zoology and Department of Life Science, National Taiwan University, Taipei, Taiwan \\ ${ }^{\mathrm{b}}$ Department of Biomechatronic Engineering, National Ilan University, No. 1 Shen Lung Rd., Sect. 1, Ilan 260, Taiwan
}

Received 2 November 2005; received in revised form 19 December 2005; accepted 2 February 2006

\begin{abstract}
A quantitative relationship between power densities of blood pressure $\left(P_{\mathrm{BP}}\right)$ and sympathetic nerve activity $\left(P_{\mathrm{SNA}}\right)$ in a low-frequency range (LF, $0.016-0.85 \mathrm{~Hz}$ ), expressed as $P_{\mathrm{SNA}}=P_{\mathrm{BP}} \times a \times 10^{b \times(\text { frequency) }}$ was proposed in pentobarbital-anesthetized rats. For evaluating the general applicability of this equation, the quantitative relationship of power density ratio $H(f)=P_{\mathrm{BP}} / P_{\mathrm{SNA}}$ across frequency was tested in a conscious state. Wistar rats were chronically instrumented with a femoral artery catheter and recording electrode around the renal sympathetic nerve. The blood pressure and renal sympathetic nerve activity were monitored both under pentobarbital anesthesia and in a conscious state. Linear regression analysis of the relationship between the frequency and logarithmic magnitude of the power density ratio in the LF range revealed excellent fit in both conditions ( $r=-0.96 \pm 0.01$ and $-0.93 \pm 0.01$ for anesthetized and conscious rats, respectively). Comparing the regression lines, rats under pentobarbital anesthesia had significantly larger values for the $y$-intercept and slope compared to rats in a conscious state ( $y$-intercepts: $0.80 \pm 0.09>0.53 \pm 0.08$; slopes: $-2.86 \pm 0.26>-1.62 \pm 0.21)$. Our results demonstrate that it is also feasible to use the weighted $P_{\mathrm{BP}}$ in LF as a quantitative index of sympathetic variability in conscious rats, but the evaluation of possible complications controlling the regression parameters is called for.
\end{abstract}

(C) 2006 Elsevier B.V. All rights reserved.

Keywords: Blood pressure; Sympathetic nerve activity; Low-frequency fluctuation; Power spectral analysis; Conscious rat

\section{Introduction}

Blood pressure (BP) and sympathetic nerve activity (SNA) are well known to be functionally coupled in order to maintain normal homeostatic function. Several lines of evidence suggest that SNA is a critical determinant of BP fluctuations in a frequency range slower than the respiration rate (Pagani et al., 1997; Furlan et al., 2000). Changes in low-frequency arterial pressure variability under conditions that prominently alter sympathetic tone have been presumed to represent proportional changes in vascular sympathetic outflow (Radaelli et al., 1994). However, controversies still

\footnotetext{
* Corresponding author. Tel.: +886 3 9357400x851; fax: +886 3 9326345.

E-mail address: mltsai@niu.edu.tw (M.-L. Tsai).
}

remain (Stauss et al., 1995; Andrew et al., 1998), and some studies further suggested that low-frequency fluctuations of the BP do not reflect sympathetic tone, but instead are related to sympathetic variability (Persson et al., 1992; Tsai et al., 1999, 2004).

Based on results from our previous studies (Tsai et al., 1997, 1999), we demonstrated that there is a quantitative relationship between power densities of $\mathrm{BP}\left(P_{\mathrm{BP}}\right)$ and SNA $\left(P_{\mathrm{SNA}}\right)$ in the low-frequency range $(\mathrm{LF}, 0.016-0.85 \mathrm{~Hz})$, expressed as $P_{\mathrm{SNA}}=P_{\mathrm{BP}} \times a \times 10^{b \times(\text { frequency })}$, where $a$ and $b$ are constants obtained from the $y$-intercept and slope values (respectively) of the regression line of logarithmic power density ratio $\left(H(f)=P_{\mathrm{BP}} / P_{\mathrm{SNA}}\right)$ across frequency. This supports the possible use of the $P_{\mathrm{BP}}$ in LF as a quantitative index of SNA variability in anesthetized rats. To further evaluate the utility of this equation, the same power density ratio of BP to SNA was assessed to determine whether it is 


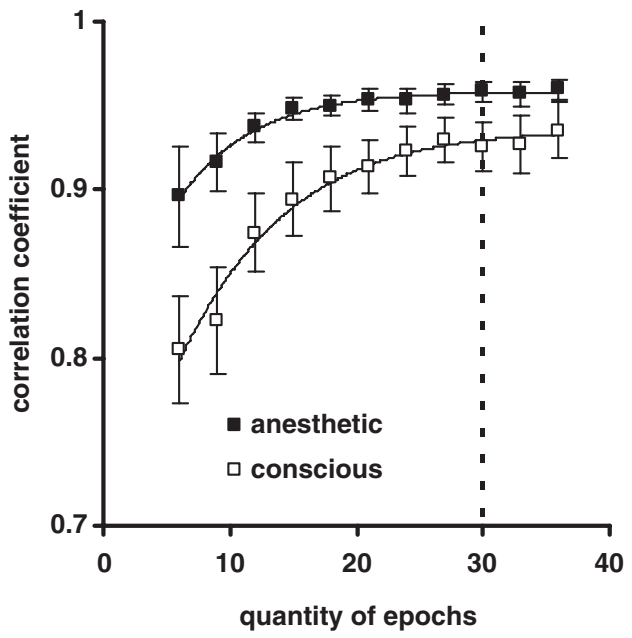

Fig. 1. Relationship of the correlation coefficient and the quantity of epochs used for averaging. The relationships in both conditions were performed with a steady high correlation coefficient when the number of epochs exceeded 30 .

also tenable in a conscious state. We recorded the BP and SNA of rats under pentobarbital anesthesia and in a conscious state. The power density ratios were calculated and the effects of pentobarbital anesthesia on the regression parameters were addressed to test the general applicability of this relationship.

\section{Materials and methods}

\subsection{Animal preparation and surgical procedures}

Seven male Wistar rats $(380-450 \mathrm{~g})$ were used. The entire experimental procedure was approved by the Institutional Animal Care and Use Committee, National Ilan University. Each rat was anesthetized with sodium pentobarbital $(50 \mathrm{mg} / \mathrm{kg}$, i.p. initially and $12.5 \mathrm{mg} / \mathrm{kg}$ i.v., supplemented as necessary during surgery). The femoral artery and vein were cannulated for systemic BP recording and drug administration. The left kidney was exposed retroperitoneally, and the renal nerve was dissected free from the surrounding connective tissues and crushed distally. A bipolar hook electrode made of stainless steel microwires (biomedical wire AS 634, Cooner Wire; Chatsworth, CA) was positioned under the renal nerve. Both the exposed nerve and electrode were encased in silicon gel (Wacker Siligel 604; Munich, Germany).

\subsection{Experimental protocol}

Following surgery, a period of time was allowed to pass to let the animal return to a lighter level of anesthesia as indicated by its ability to withdraw the hindlimb slightly when pinched. Then pentobarbital was administrated as a single dose $(12.5 \mathrm{mg} / \mathrm{kg})$ intravenously. The recording period covered approximately $40 \mathrm{~min}$ starting from the time when the BP returned to a stable level. The exact time that the rat really recovered from anesthesia was not measurable. According to a previous study by Shimokawa et al. (1998), we considered that spontaneous movement was an indicator of "awakening". The average awakening time was $182 \pm 15 \mathrm{~min}$ after the last pentobarbital administration in the 7 rats. A duration of $260-300 \mathrm{~min}$ after the last pentobarbital administration was taken as the conscious state which was approximately $1 \mathrm{~h}$ after the awakening time. The BP and renal SNA were also recorded in this period. In addition to the state of resting quietly, different tonic conditions of SNA, such as tilting, noise disturbance, or walking were included in the conscious recording. Hexamethonium bromide $(20 \mathrm{mg} / \mathrm{kg}$, i.v.) was given to determine the baseline value of renal SNA at the end of the recording session.

\subsection{Data acquisition and analysis}

The BP signal was obtained through a pressure-voltage transducer (Gould P23 ID) and amplified (Grass 7D). The electrical signal of the renal nerve was band pass-filtered at between 30 and $3 \mathrm{kHz}$ and amplified 10,000 times by a Grass P511 differential amplifier. All signals were stored on a tape recorder (model DR-886, Neuro Data, New York, NY). For offline analysis, renal nerve signals were integrated using an integrator with a 20 -ms reset time. The BP and integrated SNA signals were digitized at 1024 samples/s using an MP 100 A/D converter (BIOPAC System, Goleta, CA). Each recording period was divided into 36 epochs of $64 \mathrm{~s}$ each which did not overlap for offline analysis. According to our previous results, the averaging process produced a better fit in the linear regression analysis of the relationship between the frequency and the logarithmic power density ratio (Tsai et al., 1999). To determine the quantity of epochs for the averaging process in order to obtain a good fit for both experimental conditions, a curve relating epoch quantities with correlation coefficients was produced. The relationships in both conditions revealed steady high coefficient values when the quantity of epochs exceeded 30 (Fig. 1). Therefore, 30 epochs were used in the following regression analysis.

Spectral analysis of the integrated SNA and BP signals was carried out by fast Fourier transformation (Acknowledge version 3.5.7, BIOPAC System) after removing the DC component and applying a Hanning window. According to a previous study by Tsai et al. (2004), the power densities of each frequency in the FFT analysis were normalized by dividing them by the averaged power within the frequency band of $0.016-0.85 \mathrm{~Hz}$. The power density ratio, $H(f)$, was calculated as $H(f)=P_{\mathrm{BP}} / P_{\mathrm{SNA}}$, where $P_{\mathrm{BP}}$ and $P_{\mathrm{SNA}}$ are the respective normalized power densities of $\mathrm{BP}$ and SNA at each frequency. The $P_{\mathrm{BP}} / P_{\mathrm{SNA}}$ values at each frequency of 30 epochs were averaged for further linear regression analysis. The magnitude squared coherence function between SNA and BP was calculated using 


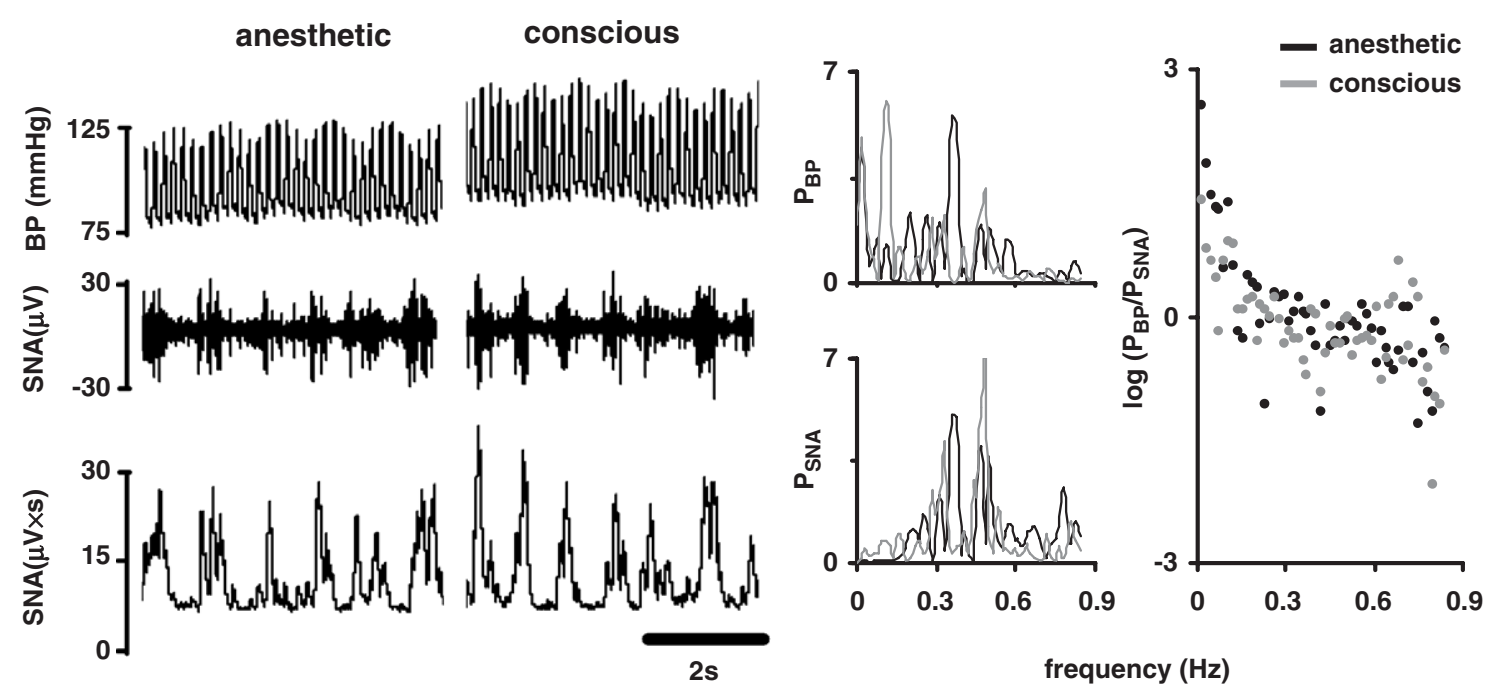

Fig. 2. A representative example of blood pressure (BP) and sympathetic nerve activity (SNA) recording, their respective spectral power (in normalized unit) and the logarithmic magnitude of power density ratio plotted against frequency under anesthetic and conscious states.

LabVIEW 6.0 software (National Instruments) and the equation of $\left|S_{x y}(f)\right|^{2} / S_{x x}(f) S_{y y}(f)$, where $S_{x y}$ is the cross spectrum and $S_{x x}$ and $S_{y y}$ are the power spectra of signals $x$ and $y$, respectively. The threshold $(=0.11)$ above which coherence exceeds zero with a significance level $\alpha=0.01$ was calculated according to the study of Barrès et al. (2004).

Paired $t$-tests were used to compare the conscious and anesthetic states in a rat. Data are expressed as the mean \pm S.E. where not specified.

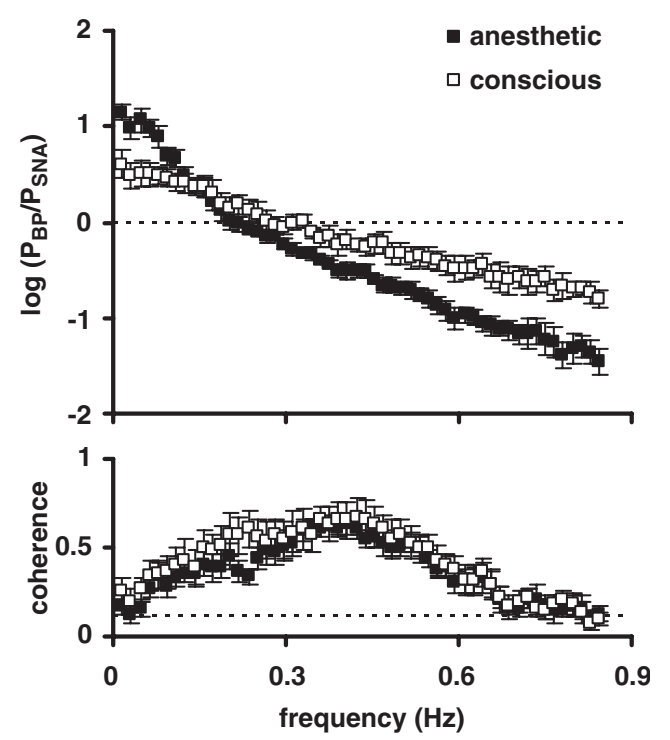

Fig. 3. The upper graph is a semi-logarithmic plots of the normalized power ratio of the BP and SNA in a frequency range lower than $0.85 \mathrm{~Hz}$. Each plot was obtained from the average of 7 rats. Note that the ratio decreased exponentially when the frequency was increased from 0.016 to $0.85 \mathrm{~Hz}$ both in anesthetized and conscious conditions. The lower graph is the squared coherence between SNA and BP. The horizontal dotted line shows the significance threshold $0.11(P<0.01)$.

\section{Results}

A representative example of $\mathrm{BP}$ and renal SNA recording, their respective spectral power and the logarithmic power density ratio plotted against frequency was shown as Fig. 2. The averaged BP readings of the recording periods of anesthesia $(87 \pm 3 \mathrm{~mm} \mathrm{Hg})$ were significant smaller $(p<0.01)$ than those in the conscious state $(101 \pm 4 \mathrm{~mm} \mathrm{Hg})$. The averaged tonic renal SNA of anesthetized and conscious states were $10.7 \pm 3.9$ and $13.2 \pm 3.3 \mu \mathrm{V} \mathrm{s}$, respectively, which did not statistically differ $(p>0.05)$. These findings were similar to the results of Shimokawa et al. (1998), who demonstrated that pentobarbital sodium $(30 \mathrm{mg} / \mathrm{kg}$, i.v. $)$ caused a decrease in the BP but no change in renal SNA.

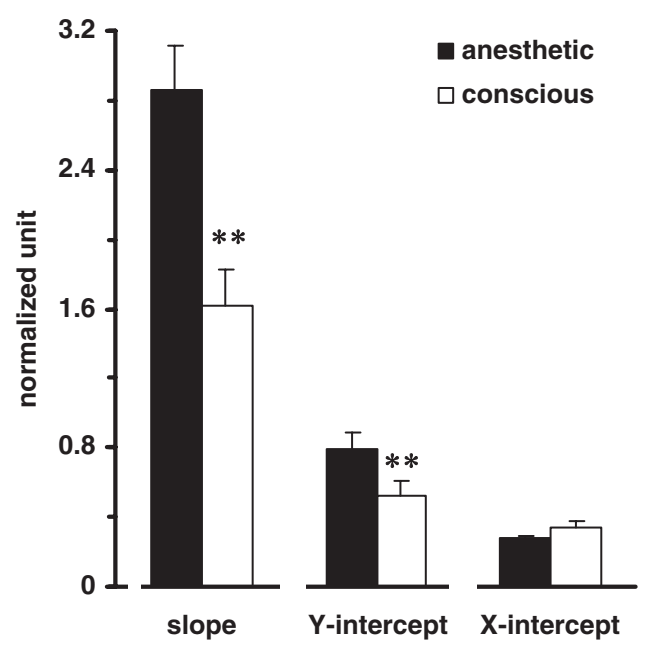

Fig. 4. Effect of pentobarbital anesthesia on the slopes, and $x$ and $y$ intercepts of the regression lines. The paired $t$-test $(n=7)$ showed significantly smaller $y$-intercept and slope values under a conscious state $(p<0.01)$. Note that the slopes are presented as absolute values. 
In both experimental conditions, the coherence exceeded the significance threshold 0.11 (Fig. 3, lower graph). The magnitude of the power density ratio decreased logarithmically with an increase in the frequency of both conditions (Fig. 3, upper graph). Linear regression analysis of the 7 rats revealed an excellent fit (with correlation coefficients of $-0.96 \pm 0.01$ and $-0.93 \pm 0.01$ for anesthetized and conscious rats, respectively). As shown in Fig. 4, the slopes of these regression lines in anesthetized and conscious states were $-2.86 \pm 0.26$ and $-1.62 \pm 0.21 ; y$-intercepts were $0.80 \pm$ 0.09 and $0.53 \pm 0.08$; and $x$-intercepts were $0.28 \pm 0.02$ and $0.34 \pm 0.04$, respectively. Rats under pentobarbital anesthesia had significantly larger $y$-intercept and slope values compared to rats in a conscious state $(p<0.01)$, but the $x$-intercept did not statistically differ $(p>0.05)$.

\section{Discussion}

It is still unknown which properties of sympatheticcardiovascular interactions contribute to the semi-logarithmic relationship. Some possibilities are that the sympathetic effect is too sluggish to mediate higher frequency variations of the BP. In a study by Malpas et al. (1999), the renal nerves of rabbits were stimulated using modulated sine patterns which varied the voltage of stimulation to between 0 and $10 \mathrm{~V}$ at a frequency of between 0.04 and $1.0 \mathrm{~Hz}$. The power of the induced oscillations in renal blood flow (RBF) was calculated using spectral analysis. The faster rhythms in stimulated SNA $(>0.6 \mathrm{~Hz})$ contributed to setting the mean level of the RBF, while only the slower rhythms $(<0.6 \mathrm{~Hz})$ induced an oscillation in the RBF. This indicated a low-pass filtering/integrating characteristic of the vasculature. Furthermore, the amplitude response of the induced oscillation generally decreased with increasing frequency of stimulation; for example, the ability of an SNA rhythm at $0.6 \mathrm{~Hz}$ to induce a rhythm in the RBF was 21 times less than that at $0.25 \mathrm{~Hz}$. Low-pass filter properties have also been described in the iliac circulation of rats, and evidence has been provided that noradrenaline reuptake mechanisms are not the frequency-limiting step of the vasculature response (Bertram et al., 2000). A study on isolated rat vascular smooth muscle cells further suggested that sympathetic modulation is limited by the $\alpha$-adrenoceptor signal transduction into muscle cells and not by an intrinsic inability of the cells to contract and relax at higher rates (Julien et al., 2001).

Under natural conditions, SNA tends to fire in bursts. This can be thought of as spontaneous central stimulation (Tsai et al., 1997). The excellent linear relationship between the frequency and the logarithmic magnitude of the power density ratio in the LF demonstrated the similar nonlinear, abruptly decreasing characteristic. For example, the $x$-intercept of the regression line was around $0.28 \mathrm{~Hz}$ under pentobarbital anesthesia, which means that the fluctuations of the 2 signals were equal in quantity at a frequency of around $0.28 \mathrm{~Hz}$. In contrast, at around $0.6 \mathrm{~Hz}$, the ratio magnitude was about -1 , which means that the spectral power of the BP was about 1/10 the power of the SNA. The fluctuation in BP coupled with an equal quantity of fluctuation in the SNA of around $0.3 \mathrm{~Hz}$ also agrees with the findings of a previous study by Brown et al. (1994), who demonstrated high coherence between fluctuations in SNA and the BP at $0.4 \mathrm{~Hz}$ both in conscious and pentobarbitalanesthetized rats. Stauss and Kregel (1996) also showed that transmission from peripheral sympathetic nerves to vascular smooth muscles is strongest in the frequency band from 0.2 to $0.5 \mathrm{~Hz}$ in conscious rats. Analysis by wavelet transformation in a simplified condition of rats (Tsai et al., 2004) also demonstrated that only the fluctuations within the frequency band of $0.25-0.4 \mathrm{~Hz}$ exhibited a good linear fit in the time domain.

Pentobarbital administration attenuates the ability of the baroreflex. In rats, light anesthesia with pentobarbital sodium $(30 \mathrm{mg} / \mathrm{kg})$ has been shown to decrease the sympathetic baroreflex gain (Shimokawa et al., 1998). Altura and Altura (1975) also demonstrated that pentobarbital dose-dependently attenuates the vascular contractions induced by epinephrine and serotonin. As shown in Fig. 4, there were statistical differences in the 2 regression parameters between the 2 conditions, with the conscious state showing a gentler slope and a lower $y$-intercept. The possibility that these regression parameters can be used to index certain sympathetic-cardiovascular interactions was implied by a simple simulating model (Cheng et al., 2004). The box and damping functions were used to simulate single fluctuations of the SNA and the corresponding BP responses, and the linearly shifted summation of each function was used to simulate simplified stimulationresponse patterns as described by Tsai et al. (1997). Power spectral analysis of these 2 simulated signals demonstrated that the similar linear regression as described for the experimental conditions and the parameters of regression line were influenced by the response intensity and the duration of the BP to SNA.

In conclusion, our results demonstrate that the $P_{\mathrm{BP}}$ in LF as a quantitative index of SNA variability can also feasibly be used in conscious rats; it is formulated as $P_{\mathrm{SNA}}=P_{\mathrm{BP}} \times a \times 10^{b \times \text { (frequency) }}$, where $a$ and $b$ are constants obtained from the $y$-intercept and slope values (respectively) of the regression line of the semi-logarithmic plot. Variations in regression parameters compared between the anesthetic and conscious states mean that the precise utility of the equation still needs to be further studied in order to evaluate possible complications from various control factors.

\section{Acknowledgements}

We thank Dr. Shaw, Fu-Zen for his programming support with coherence analysis. This study was supported by grants 
(NSC 93-2320-B-197-001 and NSC 93-2311-B002-034) from the National Science Council, Taiwan.

\section{References}

Altura, B.T., Altura, B.M., 1975. Pentobarbital and contraction of vascular smooth muscle. Am. J. Physiol. 229, 1635-1640.

Andrew, T.J., Williams, T.D., Seals, D.R., Davy, K.P., 1998. Low-frequency arterial pressure fluctuations do not reflect sympathetic outflow: gender and age differences. Am. J. Physiol. 274, H1194-H1201.

Barrès, C., Cheng, Y., Julien, C., 2004. Steady-state and dynamic responses of renal sympathetic nerve activity to air-jet stress in sinoaortic denervated rats. Hypertension 43, 629-635.

Bertram, D., Barrès, C., Cheng, Y., Julien, C., 2000. Norepinephrine reuptake, baroreflex dynamics, and arterial pressure variability in rats. Am. J. Physiol. 279, R1257-R1267.

Brown, D.R., Brown, L.V., Patwardhan, A., Randall, D.C., 1994. Sympathetic activity and blood pressure are tightly coupled at $0.4 \mathrm{~Hz}$ in conscious rats. Am. J. Physiol. 267, R1378-R1384.

Cheng, C.B., Shann, W.C., Tsai, M.L., 2004. Mathematical model for the relationship of fluctuations of blood pressure and sympathetic nerve activity. The Nineteen Joint Annual Conference of Biomedical Sciences, Taipei. p. 47. Abstract.

Furlan, R., Porta, A., Costa, F., Tank, J., Baker, L., Schiavi, R., Robertson, D., Malliani, A., Mosqueda-Garcia, R., 2000. Oscillatory patterns in sympathetic neural discharge and cardiovascular variables during orthostatic stimulus. Circulation 101, 886-892.

Malpas, S.C., Hore, T.A., Navakatykyan, M., Lukoshkova, E.V., Nguang, S.K., Austin, P.C., 1999. Resonance in the renal vasculature evoked by activation of the sympathetic nerves. Am. J. Physiol. 276, R1311-R1319.
Julien, C., Simon, C., Malpas, S.C., Stauss, H.M., 2001. Sympathetic modulation of blood pressure variability. J. Hypertens. 19, 1707-1712.

Pagani, M., Montano, N., Porta, A., Malliani, A., Abboud, F.M., Birkett, C., Somers, V.K., 1997. Relationship between spectral components of cardiovascular variabilities and direct measures of muscle sympathetic nerve activity in humans. Circulation 95, 1441-1448.

Persson, P.B., Stauss, H., Chung, O., Wittmann, U., Unger, T., 1992. Spectrum analysis of sympathetic nerve activity and blood pressure in conscious rats. Am. J. Physiol. 263, H1348-H1355.

Radaelli, A., Bernardi, L., Valle, F., Leuzzi, S., Salvucci, F., Pedrotti, L., Marchesi, E., Finardi, G., Sleight, P., 1994. Cardiovascular autonomic modulation in essential hypertension: effect of tilting. Hypertension 24, 556-563.

Shimokawa, A., Kunitake, T., Takasaki, M., Kannan, H., 1998. Differential effects of anesthetics on sympathetic nerve activity and arterial baroreceptor reflex in chronically instrumented rats. J. Auton. Nerv. Syst. 72, 46-54

Stauss, H.M., Kregel, K.C., 1996. Frequency response characteristic of sympathetic-mediated vasomotor waves in conscious rats. Am. J. Physiol. 271, H1416-H1422.

Stauss, H.M., Mrowka, R., Nafz, B., Patzak, A., Unger, T., Persson, P.B., 1995. Does low frequency power of arterial blood pressure reflect sympathetic tone? J. Auton. Nerv. Syst. 54, 145-154.

Tsai, M.L., Chu, L.W., Chai, C.Y., Yen, C.T., 1997. Frequency dependent sympathetic modulation of vasomotor tone in the anesthetized rat. Neurosci. Lett. 221, 109-112.

Tsai, M.L., Shaw, F.Z., Yen, C.T., 1999. The quantitative relationship between the fluctuations of blood pressure and sympathetic nerve activity in pentobarbital anesthetized rats. Neurosci. Lett. 263, $85-88$.

Tsai, M.L., Shann, W.C., Luo, W.R., Yen, C.T., 2004. Wavelet-based analysis of low-frequency fluctuations of blood pressure and sympathetic nerve activity in rats. Neurosci. Lett. 358 (3), 165-168. 